in the course of the natural cure. The new membrane incorporates itself with the divided edges of the cornea-just as heppens in the case of disease, when a part of the cornea is lost by ulceration or sloughing, - the iris protrudes, and a staphyloma is formed. It would be useless and hurtful to attempt any reduction after such action is set up.

Supposing, then, a case to be seen within a reasonable limit of time, what is to be done? The answer of modern ophthalmology would be this: If the prolapse occupy the circumference of the cornea, apply to the eye a drop of the fluid extract of Calabar bean, because, by the contraction of the pupil which the bean always produces, disengagement of the iris might follow. I have never been so fortunate as to get such a result. I have, too, tried the experiment when the iris has been prolapsed after the operation for tapping the anterior chambers, but in vain. If it be at the centre of the cornea, use a solution of atropine, which producing an opposite effect-dilating the pupil,--the iris might be pulled aside, and so released from the wound. Now, while I have no faith in the beneficial action of either of these in decided prolapse and strangulation, I admit their usefulness, and I speak particularly of the latter when the iris is not actually protruding and incarcerated, but just in the wound. I saw a young gentleman in less than a quarter of an hour after he had wounded the outer part of one of his corneæ with a scalpel. A portion of the iris was just embraced by the lips of the wound, to which the pupil was pulled, but did not protrude. A drop of two of a solution of a grain of atropine to two drachms of water was at once used, and in three-quarters of an hour the pupil was well dilated and perfectly round, showing that the iris was quite extricated. The dilatation was kept up for several days, till the wound was healed. A small scar on the cornea is the only trace of the accident.

Several manipulative measures are recommended. Some surgical authorities say that in no slight case of prolapse, which is seen quickly after the accident, should one abstain from attempts at reduction; and the readiest, and therefore first method, is to rub the cornea gently through the medium of the upper eyelid for a few seconds, so as to push back the iris, and then suddenly to expose the eye to a bright light. and to repeat this several times in succession, taking care that the sound eye is shaded while the friction is employed, and simultaneously exposed to the light with the injured one. I have never myself thus succeeded; neither has Dr. Mackenzie, nor did Sir Wm. Lawrence, who told me that he had made a great many trials. The plan has been recommended, no doubt, on the fact that an immediate prolapse, after the operation of extraction of a cataract, may sometimes be so reduced; but the cases are not analogous, as a moment's reflection must show.

Dr. Mackenzie thinks that, could the little bag of iris that protrudes be emptied of its aqueous humour, it would often return immediately to its place. Puncturing it would do this; but then a false pupil might be made. The method that he recommends is, instantly after dropping a solution of atropine into both eyes, to bring the patient under the influence of chloroform, and with a small curette to press on the little bag of iris, so as to empty it of its contents. The curette should enter through the wound, carrying the bit of prolapsed iris before it.

I have in recent prolapses made many trials that have been suggested, and I have arrived at the conclusion that it is better for the patient to abstain from any attempt. Such endeavours irritate the eye, and may prove fatal to it. I advise, then, that all attempt at a reduction be withbeld, and attention be directed to such treatment, generally and locally, as will subdue irritations, and prevent further damage to the eye.

A small or moderate-sized prolapse generally passes through its destined stages of repair without any ulterior result, provided ordinary care be observed during the period. My practice is to do everything to soothe the eye, and the best of all things for this is to close it with court plaster. In addition, the patient should be kept tranquil. These measures should be continued till the lachrymation and intolerance to light have ceased, when the repair is well established-a condition that will be manifested by the loss of undue vascularity in the eye, except just where the protrusion is, the vessels there being the last to disappear. But if the wound should involve a portion of the sclerotica as well as the cornea, in which case the sensitive ciliary region is involved, or if the prolapse be very large, or if it be accompanied with any other damage to the eye, any of the other effects of a blow, there is greater seriousness in the accident, and longer treatment is needed.

Some notice must be taken of the piece of displaced iris, that

without the eye. In primary prolapse the less that is done the better. I have tried all the recommendations, and carefully watched the different kinds of treatment adopted by other surgeons, and I came to the conclusion that those patients did best in which there was no irritation produced by the nitrate of silver or any other drug; they made the best recoveries. Several times $I$ have seen damage done. The solid nitrate of silver is apt to destroy the adhesions to the cornea, and thereby produce a greater prolapse. Neither do I snip away any portion of the iris ; it is unnecessary, because the soft and delicate bit never irritates, and the doing of which is itself an injury that bas to be repaired. Whatever is not needed in the formation of a proper cicatrix is sure to be efficiently and neatly removed by a natural process, and with a better result and quicker than if interfered with by art.

There is a tendency among surgeons to cease attention to these accidents too soon, and the consequence often is a renewal of the inflammation, with the attendant events.

The effects of a prolapse is according to the displacement of the pupil and its diminution. Sometimes, therefore, vision is not damaged. The more the pupil is pulled to the edge of the cornea, the more is perfect sight interfered with.

Cataract is often the direct effect of this lesion, and that, too, when neither crystalline lens nor its capsule has been injured, and it may commence in a few hours, or may not be manifested for days, weeks, or months. The treatment of the cataract should be delayed till the eye has recovered from all the inflammatory and irritating effects of the accident.

Brook-street, April, 1 is68.

\title{
ON THE MODES OF DEATH IN EPILEPSY.
}

By G. MACKENZIE BACON, M.D.,

MEDICAI SUPERINTENDENT OF THB CAMBRIDGE COUNTY ASYLUM.

THE immediate canse of death in epilepsy is a matter which is not always easily solved, and one which is not often discussed in works on medicine, most probably from lack of information. This silence on the part of writers is not to be wondered at, when one considers how few epileptics die in hospitals or under the observation of the more learned teachers of medicine ; for, as such patients are generally incurable, and also unpleasant companions, they seldom figure long as inpatients. But there is another cause far more active, and connected with the nature of the disease-viz., that, inasmuch as the mind always suffers from prolonged epilepsy, in a certain time the victims of this disease, or at all events a large proportion of them, disappear from society, and require seclusion in an asylum, where they are lost to the world.

Few, perhaps, are aware how largely epileptics figure in asylum statistics; but in these may be found most of the many failures in treatment of this disease by the general physician. Thus, taking only the pauper class (which can hardly have any special liability to epilepsy), there were on January 1st, 1867 , in the public asylums of England and Wales, 24,748 patients, of whom 22,257 were "probably incurable," and of these last 3354 were epileptic-i. e., $15^{: 5}$ per cent. of the whole number, - a large proportion to represent one cause of the total insanity. I only mention this incidentally to show that this is one of the great results of epilepsy, and that, therefore, the "intra-asylum" features of the disease form an essential element in its consideration.

My object at present, however, is to show some of the modes of death in epilepsy, and this is best illustrated by asylum experience. I am well aware how difficult it is to frame a rigid detinition of "epilepsy;" but I think a distinction must be drawn between those cases in which the convulsions are known to be due to some other disease, and those in which there is no organic disease to account for them; in other words, between "primary" and "secondary" epilepsy. Probably at some future time we shall attain to a more precise knowledge of the causes, now so obscure, of many forms of epilepsy, and shall then reduce to the minimum the primary or unexplained; but in our present ignorance we must be content to draw the line somewhere, and for this reason I shonld not include under the name of epilepsy proper, cases of convulsions in the course of general paralysis, those due to softening, or effusion on, the brain, nor those occurring in Bright's disease. It seems to me as little reasonable to merge such cases indiscriminately in one category, and consider them as positive diseases, as it would 
be to treat the feverishness of a catarrh as a special complaint. It may be difficult to express such a distinction in words, but some such method must le present to the mind in considering the various furms of eplepsy, at least if we are to study it to any useful purpose. The confusion caused by the reckless use of the word eplepsy, as explaining everything and representiog nothing, is apparent as suom as une tries to estimate the mortality due to this cause, for no one can possibly tell what is included under the word epilepsy in the mortuary hists.

If we read in the Reyistrar-General's Report for 1864 that 2406 deaths were due to epilepsy, we get no detinite idea as to the real cause of death, ancl cannut tell whether the patients died directly from the fit or in quite a different manner. Indeed, the only proper conclusion to be drawn is, that an individual who was epileptic, amongst other faults, is dead. Surely we might get a nearer approximation to truth than this. The unsatistactoriness of this surt of information is seen by the vague way in which this subject is dealt with in treatises on Epilepsy. Thus Dr. Sieveking, in his book, says-"The fit occasionally proves fatal by the exhaustion of nervous power, or by the interruption of the respiratory process; brt more frequently death results from the supervention of other diseases, or from the complications with which epilepsy is associated. The frequency of death from epilepsy bears no proportion, it appears to me, to the frequency and the importance of the disease itself ; ...... of the immediate cause of death in epilepsy we have no data.

Now asylum experience furnishes some facts of importance in elucidation of this question, and I will endeavour to point out, as concisely as possible, their bearing. Excluding, then, cases in which the fits are known to be secoudary to otr er disease, the causes of death may be classed under the following categories :-

1. Those arising from the long-continued effects of the disease on the body;

2. Deaths after a rapid succession of fits;

3. Sudden dearbs in a tit;

4. Accidents die to fits.

1. Under the first head I would include what is properly expressed by the term "the decay of epilepsy"-i.e., the condition patients reach who have had itts for years, and, after becoming demented, gralually lose the use of their limbs, and die out, wasted and exhausted, without the supervention of any structural disease. These cases are always to be fouvd in the wards of a public asylum, and in these are often discovered at the post-mortem inequalities and defects of the brain-abnormalitits only indirectly threatening hfe.

2. Some epileptics die after a series of tits lasting through two or three days, and their death may be ascribed to "epileptic coma," that being the final stage.

3. Deaths in a fit are more rare, and require the most consideration. The following are some of the causes:--(1) Asphyxia from the violence of the spasm, and consequent venous congestion. (2) Sudden loss of nervous power, due, probably, to the state of the heart or its nerves, and not occurring in young persons. (3) Suffication: which may arise from $(\alpha)$ the face being buried in the pillow (or any soft material-mud, for instance) by the patient turning over in a fit; (b) from the impaction of food in the larynix or asophagus; $(c)$ from regurgitation of liquids from the stomach into the lungs, or the flooding of the lungs with blood from a vessel giving way during a fit, as happened to a phthisical man with a vomica.

4. Accidents may include a great variety of modes of death, but I only refer to those directly connected with the fit, such as when a patient falls in a fit and fractures his skull, or receives other injuries from which he dies; or when such an incident occurs as the following, recorded at the Notts Asylum: "An epileptic patient was found early in the morning dead, with his head on the floor and his legs on the bed, twisted among the bedclothes, which retained him in this unfavourable position. On inquiry, it appeared that he had been seized with a fit, had rolled thus partially out of bed, and expired."

I have condensed these observations as much as possible, wishing merely to point out some of the ascertained causes of death; but I am prepared to llustrate each of the classifications I have marle by cases oceurring within my own experience or recorded by or hers. Some of the instances are curious accidents, and in most the true cause of death could hardly he properly determined without a post-mortem examination. Of the causes enunierated, that of suffocation in bed is far from uncommon in asylums (five cases having occurred in a large asylum in one year); but this does not seem to be recognised as such in the outer world. I hope to enter more fully on the whole subject at a future time, but I think 1 bave even uow furnished some of those "data" sail to be wanting; and, mureover, that if practitioners would adopt some such system in recording deaths from epilepsy we should not have to lament such a meaningless blank as the word now represents in lists of mortality.

Fulbourn, near Cambridge, April, 1868.

\section{EXPERIMENTS WITH THE POISON OF THE COBRA DI CAPELLA.}

\section{By JOHN SHORTT, M.D., F.L.S., M.R.C.P.L., \&c.,} SUPERLNTENDENT-GENERAL OF PACCLIY, MADRAS PRKSIDENCX.

THw venom of the cobra is contained in two sacs or pouches; one on each side of the upper jaw of the reptile, and a mov-. able tooth or fang is connected with each pouch. The fang is curved, grooved along its convex surface, and with an opening at the free extremity, which terminates in a sharp point. The fangs are very strong, and the average thickness of each is from one-twelfth to one-sixteenth of an inch. In the natural state the fangs lie horizontally; but when the animal is excited the cobra raises its head, expands its hood, and the fangs become erect, or nearly so, ready for action. Frequently in. this attitude it may strike at an enemy, and wound, so as to draw blood, without injecting any poison. To effect this it darts its head straight lorward, seizes some part between its jaws, and, thus grasping it, the poison is ejected into the wound, contaninating its whole extent from top to bottom, which is done by the poison escaping from an opening at the base of the convex surface of the fang, and descending along the upper surface of the groove, as well as through it in the opening at the apex; the poisun passing through the former channel contamuating the upper side, and that coming through the latter the bottom of the wound.

Having inflicted the wound and ejected the poison, the cobra immediately, and as if instinctively aware of having done its work, loosens its grasp, and, to extricate the curvature of the fangs, turns its bead slightly to one side, and thus, without injury to them, withdraws buth. The venom is caused to exude by the compression of a powerful pair of muscles, one on either side of the jaw.

The moment the venom is injected by the cobra into wound, sharp pain appears to be caused in the part, as evidenced by the subjects of experiments beginning to wince and howl, and becoming lame almust immedrately if any of the extremities are wounded. The poison seems to be immediately taken up by the venous radicles and carried into the circulation; and, according to the size and vigour of the animal the size and activity of the cobra, as well as the concentrated state in which the poison-secretion may be in the sacs, death is the result in from five minutes to twelve hours.

The following experiments bear upon this.

\section{Experiment No. 1.}

March 11th, 1867.-A full-grown pariah dog was bitten by a large cobra, measuring five feet in length, at 11.45 A.M., on the inner part of the left thigh. Two blood points became visible, indicating the situation of the wound, and the dog immediately drew up the leg and walked lame. The animal frequently looked at the wounded part, and was inclined to lick the part with its tongue. Convulsions set in at 11.58 , it micturated, the tongue protruded from the mouth, and the animal quietly expired at $1.3 \%$. Death in $1 \mathrm{~h}$. $18 \mathrm{~min}$.

Sectio cadaveris, two hours after deatk. - Head carefully opened. There was congestion of the veins, which contained dark, jelly-like blood; and the several siuuses were distended with blood of a similar kind. A trace of effusion of serum was evidenord by thickening of the pia mater from serous exudation. Brain of natural form and consistence. The puncta vasculosa were dark-coloured. The lateral ventricles contained about two drachms of serum of a slightly reddish tinge.Spinal column: On opening this canal the vertebral veins were at once seen to be distended and of a dark-blue colour. On opening these they were found filled with the same dark, jelly.like bluod. There was some slight congestion, and indications of slight serous exudation within the membranes. The spinal cord itself was normal. - Thorax: The lungs filled the cavity of the thorax, and were not congested except at the base, which looked somewhat florid. The right cavities of the heart con- 